\title{
Neutrophil plasticity
}

New research published in Cancer Cell suggests that tumour-associated neutrophils can have antitumorigenic ('N1') or protumorigenic ('N2') functions, a plasticity that has been well described for M1 and M2 macrophage subsets. It is suggested that the presence of transforming growth factor- $\beta$ (TGF $\beta$ ) in the tumour microenvironment prevents the generation of N1 neutrophils and that overriding this inhibition by blockade of TGF $\beta$ contributes to the known antitumour effects of TGF $\beta$ blockers.

Blockade of the immunosuppressive cytokine TGF $\beta$ inhibits tumour growth in several ways, for

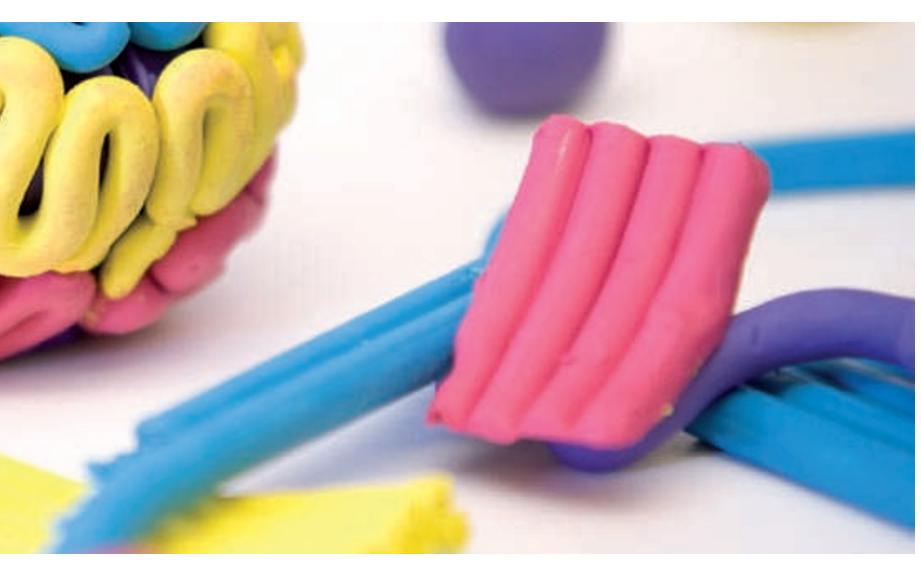

example by allowing the activation of $\mathrm{CD}^{+} \mathrm{T}$ cells and macrophages. The finding by Fridlender et al. that large numbers of neutrophils infiltrate the tumours of mice treated with the TGF $\beta$ blocker SM16 led to the suggestion that neutrophils are also involved in the antitumour effects of TGF $\beta$ blockade. Neutrophils accumulated in the tumours of SM16-treated mice in response to increased production of chemoattractants, probably produced by tumour-associated macrophages, and increased expression of adhesion molecules that support neutrophil recruitment. Depletion of the infiltrating neutrophils using a neutrophilspecific antibody in SM16-treated mice significantly reduced the antitumour effect of SM16, suggesting that tumour-associated neutrophils in SM16-treated animals have antitumoral activity.

Further analysis indicated that only tumour-associated neutrophils isolated from SM16-treated mice - not neutrophils from untreated mice could directly kill tumour cells in vitro, possibly as a result of increased expression of the death receptor CD95 and increased production of oxygen radicals. Moreover, the phenotype of neutrophils from SM16treated tumours was consistent with immunostimulatory activity, such as lower levels of arginase (an immunosuppressant) and higher levels of tumour necrosis factor (an immune stimulator) than neutrophils from untreated tumours.

Finally, the authors showed that depletion of neutrophils in untreated mice led to increased $\mathrm{CD}^{+} \mathrm{T}$ cell activation and decreased tumour growth. By contrast, depletion of neutrophils from SM16-treated mice decreased SM16-induced CD8 ${ }^{+} \mathrm{T}$ cell activation and antitumour effects, which supports the idea that during TGF $\beta$ inhibition neutrophils assume a tumour-cytotoxic N1 phenotype and support $\mathrm{CD}^{+} \mathrm{T}$ cell activation to inhibit tumour development.

More work will be required to determine to what extent this neutrophil plasticity mirrors macrophage plasticity.

Lucy Bird

ORIGINAL RESEARCH PAPER Fridlender, Z. G. et al. Polarization of tumor-associated neutrophil phenotype by TGF- $\beta$ : "N1" versus "N2" TAN. Cancer Cell 16, 183-194 (2009) 